\section{Hypothesis for origin of planktonic patchiness}

Although environmental heterogeneity can contribute to the observed patchiness in oceanic planktonic populations, biological interactions between phytoplankton and herbivorous copepods can lead to similar patterns. The phenomenon of spontaneous pattern generation even in a homogeneous environment through the interplay of reaction and movement is a very general property of the class of mathematical 'reaction-diffusion' equations used to model such systems, and there are several ways in which such patterns may occur. The result may be either spatiotemporal patterns ${ }^{1}$ or timeindependent spatial patterns. Usually one thinks of diffusion as damping inhomogeneities, and a hypothesis put forward by Steele $^{2}$ essentially relies on a balance reached between the dehomogenising aspects of local interaction and the homogenising influence of diffusion to produce pattern. On the other hand, it has also been suggested ${ }^{3-6}$ that in some conditions diffusion can destabilise an otherwise stable interaction (in the manner originally suggested by Turing ${ }^{7}$ ) to produce pattern. We have explored ${ }^{5}$ this further in a nonlinear analysis that describes the final stages of pattern formation, and the resultant calculations provide some insights into the spatial scale of patterns which might arise in this way.

The results that we describe depend on two hypothesised properties of the full equations: an autocatalytic effect in phytoplankton density which is attributed to a reduced efficiency of herbivory as phytoplankton density increases (a Holling ${ }^{8}$ Type 2 functional response), and differential dispersal rates which favour higher herbivore motility. Both assumptions may be justified on theoretical grounds (the work of Holling for the first, and the greater mobility of herbivores by comparison with phytoplankton for the second), but the data are at present. insufficient to provide the real test.

The relevant equations ${ }^{5}$ thus take the form

$$
\begin{aligned}
\partial P / \partial t & =a P+e P^{2}-b P H+\nabla \cdot(\mu \nabla P) \\
\partial H / \partial t & =c P H-d H^{2}+\nabla \cdot(v \nabla H)
\end{aligned}
$$

where $P$ is phytoplankton density, $H$ herbivore density, $t$ time, and $\mu$ and $v$ are species-specific diffusion coefficients. $a, b, c, d$ and $e$ are all assumed positive. The assumption of passive diffusion is made for simplicity only; more complicated movement patterns can also lead to diffusive instability.

Assuming homogeneity of dispersal rates and restricting attention to one space dimension, we obtain the modified equations

$$
\begin{aligned}
& \partial P / \partial t=a P+e P^{2}-b P H+\mu\left(\partial^{2} P / \partial x^{2}\right) \\
& \partial H / \partial t=c P H-d H^{2}+v\left(\partial^{2} H / \partial x^{2}\right)
\end{aligned}
$$

In the linearised study of small disturbances, one exposes the system to small amplitude perturbations of the initial form

$$
P=c_{1} \cos q x, H=c_{2} \cos q x
$$

(Rather general disturbances can be Fourier synthesised from such perturbations.)

The system is seen to have a spatially uniform stable equilibrium

$$
P=(a d) /(b c-e d), H=(a c) /(b c-e d)
$$

provided $b c-e d>0, c>e$, and

$$
R=v / \mu<[1 /\{\sqrt{ }(\mathrm{b} / \mathrm{d})-\sqrt{ }[(b / d)-(e / c)]\}]^{2}=R_{\mathrm{cr}}
$$

We note that $R_{\mathrm{cr}}>1$.
When $R>R_{\mathrm{c} r}$, the local activation effect due to the autocatalytic term interacts with the long range inhibition ${ }^{9}$ to destabilise the uniform state. For $R$ slightly greater than $R_{\mathrm{cr}}$, a perturbation will serve to destabilise if its initial wavelength is approximately equal to $1 / q_{\mathrm{cr}}$, where

$$
q_{\mathrm{cr}}=[\sqrt{ }(b / d) / \sqrt{ }[(b / d)-(e / c)]]-1
$$

By a combination of successive approximations and multiple time scales, we have shown ${ }^{5}$ that for $R$ slightly greater than $R_{\text {cr }}$, the uniform state is replaced by a new steady state in which plant and herbivore are more concentrated in certain regions (with an overall elevation in mean herbivore density). The limit to the growth of the perturbation is a result of the distortion of the initial periodic spatial distribution due to nonlinear interactions.

When two-dimensional effects are introduced, we expect (as happens in the discrete spatial case) that perturbations of large enough amplitude will be shown to destablise systems which are stable to small perturbations, and that generally the new steady state which arises from instability of the uniform state will either be net-like or spot-like, depending on a certain combination of parameters. This type of analysis is valid for initially homogeneous regions that are large compared with $1 / q_{\mathrm{cr}}$.

We emphasise that we offer here only one mechanism for generating pattern-not necessarily the principal one. In eventually distinguishing between hypotheses, however, it should be helpful to do the kind of nonlinear analysis we have carried out. To our knowledge this has not previously been done for any of the hypotheses.

L.A.S. was supported by the Army Research Office and S.A.L. by the National Science Foundation. The research was also supported by the US-Israel Binational Science Foundation.

Simon A. LeVIN

Section of Ecology and Systematics

and Department of Theoretical and Applied Mechanics,

Cornell University.

Ithaca, New York 14853

Department of Applied Mathematics,

Weizmann Institute of Science,

Rehovot, Israel

and Rennselaer Polytechnic Institute,

Troy, New York 12181

Received July 31, 1975 ; accepted January 5, 1976.

1 Kopell, N., and Howard, L. N., Lectures on Mathematics in the Life Sciences, 7 (edit. by Levin, S. A.), 201-216 (American Mathematical Society, Providence, Rhode Island, 1974).

Steele, J. H., Coastai Upwelling Ecosystems Analysis Newsletter, 2(4), 3-7 (1973); Nature, 248, 83(1974).

Levin, S. A., Am. Nat., 108, 207-228 (1974)

(1972).

Segel, L. A., and Levin, S. A., Top. stat. Mech. Biophys. Proc. AIP Conf., 27 (American Institute of Physics, in the press).

O A A Baltimore, 1974).

Turing, A., Phil. Trans, R. Soc., B237, 37-72 (1952).

8 Holling, C.'S., Mem. entom. Soc. Can., 45, 1-60 (1965).

9 Gierer, A. and Meinhardt. H. Lectures on Mathematics in the Life Sciences, 7 (edit by Levin, S. A.), 163-183 (American Mathematical Society, Providence, Rhode Island, 1974).

\section{Intercalary regeneration in imaginal wing disk of Drosophila melanogaster}

IN this paper we show for the first time that the developmental capacity of imaginal disk cells from Drosophila can be altered by appropriate grafting operations. The appendages of amphibians and immature arthropods, and the imaginal disks of holometabolous insects, are capable of undergoing pattern regulation when parts are removed. (Pattern regulation is used here to describe the alteration of cell fates in response to an 